\title{
A Systematic Review and Meta-Analysis of Long-Term Development of Early Term Infants
}

\author{
Ying Dong ${ }^{\mathrm{a}}$ Shao-jie Chen ${ }^{\mathrm{b}}$ Jia-lin Yu ${ }^{\mathrm{a}}$ \\ a Department of Neonatology, Children's Hospital of Chongqing Medical University, Ministry of Education Key \\ Laboratory of Child Development and Disorders, Key Laboratory of Pediatrics in Chongqing and Chongqing \\ International Science and Technology Cooperation Center for Child Development and Disroders, and \\ ${ }^{b}$ Department of Cardiology, The 2nd Affiliated Hospital of Chongqing Medical University, Chongqing, China
}

\section{Key Words}

Early term infants $\cdot$ Developmental outcomes .

Neurodevelopment

\begin{abstract}
Background: Births between 37 0/7 and 386/7 weeks of gestation are newly defined as early term infants (ETIs), and are increasingly considered to be at higher risk of adverse outcomes than infants born at 39-41 weeks' gestation. To date, the long-term development of ETIs has not been systematically reviewed. Objective: To assess the effect of being born early term on long-term developmental outcomes. Methods: The literature of MEDLINE, EMBASE, Cochrane Database of Systematic Reviews and Cochrane Central Register of Controlled Trials, and abstracts from the Society of Pediatric Research were searched. If two or more studies regarding the same outcome were retrieved, a meta-analysis was conducted by RevMan 5. A sensitivity analysis was performed to assess the heterogeneity. Results: Eleven studies involving 4 categories of long-term development were included. Compared with infants born at 39-41 weeks' gestation, ETIs had poorer outcomes in school performance, neurodevelopment, behavior and emotional status and long-term social outcomes. Meta-analyses showed that being born early term significantly increased the risk of cerebral palsy [relative risk, RR, 1.75 (95\% confidence interval, $\mathrm{Cl}, 1.32,2.31)]$ and
\end{abstract}

mathematical difficulties [RR $1.13(95 \% \mathrm{Cl} 1.04,1.21)]$. The statistical test of heterogeneity for cerebral palsy was significant. Sensitivity analysis demonstrated that variations in follow-up periods were associated with heterogeneity. Conclusion: Emerging evidence suggests that ETIs are at risk of adverse long-term outcomes. Due to paucity and heterogeneity of the existing data, future research is needed to clarify the long-term risk of being born early term.

Copyright $\odot 2012$ S. Karger AG, Basel

\section{Introduction}

Historically, gestation has been treated as a binary factor, with 37 gestational weeks defined as the threshold of fetal maturity [1]. Compared with term infants (37 0/7 to 41 6/7 gestational weeks), neonates delivered before 37 weeks are associated with a significantly higher risk of morbidity and mortality, and the risk increases with increasing degree of preterm birth [2]. Infants born at term, on the other hand, are considered healthy and treated as a homogeneous group with respect to neonatal outcomes. However, emerging evidence shows that term births prior to 39 gestational weeks are more likely to have adverse outcomes than those after 39 weeks, indicating gestation is a biologic continuum and the dose-effect exists even within the term spectrum [3]. In the last decade, births

\section{KARGER}

Fax +41613061234 E-Mail karger@karger.ch www.karger.com
(C) 2012 S. Karger AG, Base

$1661-7800 / 12 / 1023-0212 \$ 38.00 / 0$

Accessible online at:

www.karger.com/neo
Dr. Jia-lin Yu

Department of Neonatology, Children's Hospital of Chongqing Medical University

No. 136 Zhongshan er Road, Yuzhong District

Chongqing 400014 (China)

Tel.+86 1389606 9217, E-Mail yujialin486@ sohu.com 
occurring between 37 0/7 and 38 6/7 weeks, newly defined as early term infants (ETIs), increased by nearly $20 \%$, while the delivery of late preterm infants (340/7 to $366 / 7$ weeks) increased by $12 \%$ and births after 40 gestational weeks have declined $[1,4]$. ETIs are the fastest growing subgroup of neonates, and account for $17.5 \%$ of the annual live births in the United States, whereas preterm births constitute approximately $12.5 \%$ in the same cohort $[5,6]$. Data from other regions in the world are largely unknown due to the general unawareness of infants born early term. From a public health perspective, ETIs are the main target of health services because the majority of adverse outcomes are attributable to this special subgroup of neonates.

The negative effect of being born early term on shortterm morbidity and mortality has been well documented $[3,6]$. Although ETIs have a significantly higher risk of morbidity and are more likely to die in infancy when compared with term neonates born after 39 gestational weeks, an increasing number of infants born early term can survive into adolescence or adulthood on account of the progress in neonatal care [3]. In contrast to the abundance of information on short-term outcomes, there is a dearth of research relating to the long-term development of ETIs, and this problem has not been systematically evaluated as far as we are aware. Addressing this issue will help optimize management of a majority of neonates and improve general public welfare. In light of the fact, we undertook a systematic review to explore the longterm effect of being born early term on development.

\section{Method}

\section{Search Strategy}

A list of literature was identified by searching electronic databases including MEDLINE (1980-2012), EMBASE (1980-2012), Cochrane Database of Systematic Reviews (the Cochrane Library, issue 1,2012) and Cochrane Central Register of Controlled Trials (CENTRAL, the Cochrane Library, Issue 1, 2012) in January, 2012. The following terms were used for searching: early term, or 37 to 38 weeks, and long-term outcome, development, neurodevelopment, child development, health, cognitive, motor, behavior, attention deficit, intelligence, and school. No language restriction was applied. Additionally, the abstract books from the Society of Pediatric Research from 1998 onwards were manually searched during the same time. The reference list of identified studies and reviews were sought for additional citations.

\section{Data Extraction}

Retrieved articles were assessed for eligibility. To be included in the study, the participants had to be ETIs (37 0/7 to $386 / 7$ gestational weeks). The control group (term infants born after 38 completed gestational weeks) was defined; long-term developmental outcomes of both ETIs and infants in the control group were reported; data and research methodology were adequately described. Studies reporting outcomes by week of gestation were also deemed eligible as long as term births were included. Reviews and follow-up studies on a certain preexisting disorder (e.g. small for gestational age, SGA) that would possibly increase the likelihood of a compromised developmental outcome were excluded. Two reviewers searched literature independently, and discrepancies were resolved by discussions until consensus was achieved. Data on authors, study location, study design, participants, age at assessment and outcomes were tabulated. Where data were incomplete, the corresponding author was contacted for further information.

\section{Methodological Quality of the Studies}

Included were non-randomized prognostic studies aimed at investigating long-term outcomes of infants. In light of limited information on the appraisal of such studies, we performed the quality assessment by referring to previous studies [7]. Hayden's framework and the International Society for Pharmacoeconomics and Outcomes Research retrospective database checklist were used to assess the following 7 areas of potential bias: data source, study participation, study attrition, prognostic factor measurement, confounding measurement and account, outcome measurement and analysis $[8,9]$. An ultimate appraisal of yes, partly, no or unsure was given to each area after generally assessing a list of quality items relevant to it [8]. This type of quality assessment tool is recommended against a score scale because scales are considered to reduce scientific judgment and are more likely to include criteria that do not directly relate to study validity $[8,10]$. Two reviewers assessed the quality of studies independently. Kappa value was used to evaluate the inter-rater agreement.

\section{Statistical Analysis}

Outcomes of included studies were divided into the following 4 categories: neurodevelopment, schooling problems, behavior and emotional status, and long-term social outcomes. Relative risk (RR) and 95\% confidence interval (CI) were calculated for dichotomous outcome variables. Continuous outcome variables were expressed as mean difference and 95\% CI. If two or more studies regarding the same outcome were retrieved, a meta-analysis was conducted by using RevMan 5. If appropriate, a fixed effect model was used for combining results in the meta-analysis. Heterogeneity was defined as a significant test of $\mathrm{p}<0.1$, and the $\mathrm{I}^{2}$ statistic was used to estimate the heterogeneity. In addition, a sensitivity analysis was carried out to assess the heterogeneity. On the other hand, a descriptive methodology was chosen and a narrative synthesis was performed for studies with irrelevant outcomes.

\section{Results}

\section{Included Studies}

The initial search yielded 1,671 potential related citations. After excluding reviews, irrelevant studies and studies not reporting on ETIs, a total of 12 studies were 
Fig. 1. Selection process.

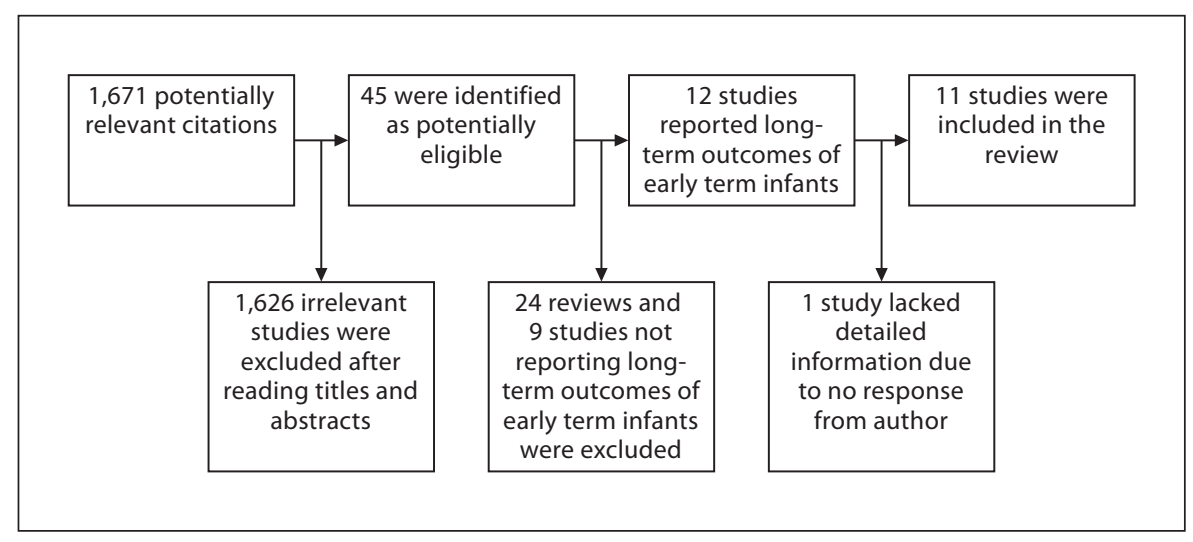

identified. Attempts were made to contact authors of 2 studies for further information $[11,12]$. One of them (Dr. Mathiasen) gave a response and the other failed to be contacted. Finally 11 eligible studies were included and the selection process is detailed in figure 1 . Characteristics of the 11 studies are summarized in table 1 and quality assessment is presented in table $2[11,13-22]$. None of the 11 studies focused solely on ETIs, and all of them included a subgroup of infants born at 37-38 gestational weeks within the study population. Most of the included studies showed an overall low level of risk for bias. The quality assessment demonstrated high concordance between the 2 raters as measured by kappa value $(\kappa=0.741 ; \mathrm{p}<0.0001)$, indicating there were no major systematic disagreements. A summary of the results of included studies were shown in table 3.

\section{Neurodevelopment}

Neurodevelopmental disabilities have been defined as 'a group of heterogeneous conditions that share a disturbance in the acquisition of basic developmental skills in a chronologically appropriate manner [23].' Cerebral palsy (CP) and intellectual disability were included in this broad definition. Data for CP were reported in 3 studies [11, 17, 22], and a random-effects meta-analysis showed that the pooled risk of CP was 1.75 (95\% CI 1.32, 2.31) times higher for ETIs than infants born at 39-41 weeks' gestation (fig. 1). However, the $\chi^{2}$ test for heterogeneity was significant $\left(\chi^{2}=20.38, p<0.0001\right)$. Among the 3 studies, Moster et al. [22] showed the lowest risk of CP for ETIs, which could be explained by the fact that their data are retrieved by using a disability register, and only children surviving to 4 years old are included, thus fewer patients may be included. Heterogeneity was no longer significant after excluding the data reported by Moster et al. [22] from this meta-analysis, and the pooled risk of CP was 1.99 (95\% CI $1.79,2.22)$, suggesting that the follow-up period is a factor explaining the heterogeneity. There were 3 studies that focused on the impact of being born early term on intelligence scores $[13,18,21]$. However, the data were not eligible for quantitative syntheses due to the widely varying scales used for measurement. It is noteworthy that the score gap between early term group and control group narrowed down when confounding factors like maternal education and family socioeconomic status (SES) were adjusted [13, 21]. Ekeus et al. [18] showed that accounting for perinatal factors such as SGA would further dilute the association between gestation and intelligence scores.

\section{Schooling Problems}

Schooling problems have been broadly defined as the need for special education, education below age level, special support in regular school, or poor performance in comparison with pupils in the same class [24]. The 5 studies relating to schooling problems were highly variable with respect to the population size, demographic factors, outcomes and follow-up periods [11, 14-16, 19]. Two of the 5 studies reported data on difficulties in requiring mathematical skills and were subjected to meta-analysis $[14,15]$. We noted that the risk of having mathematical difficulties for ETIs was not significant in Kirkegaard's study [RR 0.82 (95\% CI $0.42,1.6)$ ], which could be explained by the small population size and a low reporting rate [15]. However, the pooled result showed a significantly higher risk of having mathematical difficulties among early term group than their controls [RR 1.13 (95\% CI $1.04,1.21)]$ with no significant heterogeneity between the studies $\left(\mathrm{I}^{2}=0 \% ; \mathrm{p}=0.35\right)$, indicating the power of detecting an effect was increased after data combination (fig. 2). It can be seen from table 3 that after taking a series of 
Table 1. Description of included studies

\begin{tabular}{|c|c|c|c|c|c|c|c|c|}
\hline \multirow[t]{2}{*}{ Study } & \multirow[t]{2}{*}{ Location } & \multirow[t]{2}{*}{ Study design } & \multirow{2}{*}{$\begin{array}{l}\text { Birth of } \\
\text { cohort } \\
\text { year }\end{array}$} & \multirow{2}{*}{$\begin{array}{l}\text { Follow-up } \\
\text { years }\end{array}$} & \multicolumn{2}{|c|}{ Description of infants } & \multirow[t]{2}{*}{ Exclusions } & \multirow[t]{2}{*}{ Outcome definitions } \\
\hline & & & & & study & control & & \\
\hline $\begin{array}{l}\text { Mathiasen } \\
\text { et al. [11] }\end{array}$ & Denmark & $\begin{array}{l}\text { Retrospective cohort } \\
\text { population based }\end{array}$ & $\begin{array}{l}1988- \\
1989\end{array}$ & $18-19$ & $\begin{array}{l}37-38 \text { weeks } \\
\text { GA } \\
n=16,586\end{array}$ & $\begin{array}{l}\text { 39-41 weeks } \\
\text { GA } \\
n=84,560\end{array}$ & $\begin{array}{l}\text { BW above } 6 \text { SD or less } \\
\text { than }-6 \mathrm{SD}\end{array}$ & $\begin{array}{l}\text { (1) Exam performances } \\
\text { after the } 9 \text {-year } \\
\text { compulsory school; }(2) \\
\text { cerebral palsy diagnosed } \\
\text { by ICD- } 8 \text { and ICD-10 }\end{array}$ \\
\hline $\begin{array}{l}\text { Eide et al. } \\
{[13]}\end{array}$ & Norway & $\begin{array}{l}\text { Retrospective cohort } \\
\text { population based }\end{array}$ & $\begin{array}{l}1967- \\
1979\end{array}$ & 18 & $\begin{array}{l}37-38 \text { weeks } \\
\text { GA men } \\
n=37,487\end{array}$ & $\begin{array}{l}\text { 39-41 weeks } \\
\text { GA men } \\
n=209,191\end{array}$ & $\begin{array}{l}\text { Permanent } \\
\text { disability }\end{array}$ & $\begin{array}{l}\text { Intellectual performance } \\
\text { by the Norwegian draft } \\
\text { revised in } 1962 \text { including } \\
\text { verbal analogues, } \\
\text { number series and } \\
\text { geometric figures }\end{array}$ \\
\hline $\begin{array}{l}\text { Quigley } \\
\text { et al. [14] }\end{array}$ & UK & $\begin{array}{l}\text { Retrospective cohort } \\
\text { population based }\end{array}$ & $\begin{array}{l}2000- \\
2001\end{array}$ & 5 & $\begin{array}{l}37-38 \text { weeks } \\
\text { GA } \\
n=1,956\end{array}$ & $\begin{array}{l}\text { 39-41 weeks } \\
\text { GA } \\
n=5,407\end{array}$ & $\begin{array}{l}\text { Implausible BW } \\
\text { for GA }\end{array}$ & $\begin{array}{l}\text { School performances } \\
\text { measured by the } \\
\text { foundation stage profile } \\
\text { assessing six areas of } \\
\text { learning }\end{array}$ \\
\hline $\begin{array}{l}\text { Kirkegaard } \\
\text { et al. [15] }\end{array}$ & Denmark & Prospective cohort & $\begin{array}{l}1990- \\
1992\end{array}$ & 10 & $\begin{array}{l}37-38 \text { weeks } \\
\text { GA } \\
n=633\end{array}$ & $\begin{array}{l}39-40 \text { weeks } \\
\text { GA } \\
n=3,081\end{array}$ & Loss to follow-up & $\begin{array}{l}\text { Skills in reading, spelling } \\
\text { and math assessed by } \\
\text { questionnaires } \\
\text { completed by parents } \\
\text { and teachers }\end{array}$ \\
\hline $\begin{array}{l}\text { MacKay } \\
\text { et al. [16] }\end{array}$ & UK & $\begin{array}{l}\text { Retrospective cohort } \\
\text { population based }\end{array}$ & $\begin{array}{l}1986- \\
2001\end{array}$ & $4-19$ & $\begin{array}{l}37-38 \text { weeks } \\
\text { GA } \\
n=71,403\end{array}$ & $\begin{array}{l}\text { 39-41 weeks } \\
\text { GA } \\
n=297,000\end{array}$ & $\begin{array}{l}\text { Maternal height }<100 \\
\mathrm{~cm} \text { or }>200 \mathrm{~cm}, \mathrm{BW} \\
<400 \mathrm{~g} \text { or }>5,000 \mathrm{~g} \text { and } \\
\text { multiple births }\end{array}$ & $\begin{array}{l}\text { Special educational } \\
\text { needs of school age } \\
\text { children }\end{array}$ \\
\hline $\begin{array}{l}\text { Lindström } \\
\text { et al. [17] }\end{array}$ & Sweden & $\begin{array}{l}\text { Retrospective cohort } \\
\text { population based }\end{array}$ & $\begin{array}{l}1987- \\
2000\end{array}$ & $6-19$ & $\begin{array}{l}37-38 \text { weeks } \\
\text { GA } \\
n=214,475\end{array}$ & $\begin{array}{l}39-41 \text { weeks } \\
\text { GA } \\
n=813,606\end{array}$ & $\begin{array}{l}\text { At least } 1 \text { significant } \\
\text { malformation, BW } \\
\text { above } 3 \text { SD or less than } \\
-6 \text { SD }\end{array}$ & $\begin{array}{l}\text { (1) ADHD medication } \\
\text { with ATC-codes } \\
\text { NO6BA01- NO6BA04 or } \\
\text { NO6BA01; (2) cerebral } \\
\text { palsy was defined as at } \\
\text { least } 1 \text { hospital } \\
\text { admission after } 2 \text { years } \\
\text { old with a diagnosis of } \\
\text { ICD-10 }\end{array}$ \\
\hline $\begin{array}{l}\text { Ekeus et al. } \\
{[18]}\end{array}$ & Sweden & $\begin{array}{l}\text { Retrospective cohort } \\
\text { population based }\end{array}$ & $\begin{array}{l}1973- \\
1976\end{array}$ & $18-19$ & $\begin{array}{l}37-38 \text { weeks } \\
\text { GA men } \\
n=19,146\end{array}$ & $\begin{array}{l}\text { 39-41 weeks } \\
\text { GA men } \\
n=94,812\end{array}$ & $\begin{array}{l}\text { BW above } 3 \text { SD or less } \\
\text { than }-6 \text { SD }\end{array}$ & $\begin{array}{l}\text { Intellectual test scores } \\
\text { measured by 'Enlistment } \\
\text { Battery } 80 \text { ' representing } \\
\text { logical, spatial, verbal } \\
\text { and technical capabilities }\end{array}$ \\
\hline $\begin{array}{l}\text { Lindström } \\
\text { et al. [19] }\end{array}$ & Sweden & $\begin{array}{l}\text { Retrospective cohort } \\
\text { population based }\end{array}$ & $\begin{array}{l}1973- \\
1979\end{array}$ & $23-29$ & $\begin{array}{l}37-38 \text { week } \\
\text { GA } \\
n=68,541\end{array}$ & $\begin{array}{l}39-41 \text { weeks } \\
\text { GA } \\
n=431,656\end{array}$ & $\begin{array}{l}\text { At least } 1 \text { reported } \\
\text { malformation, BW } \\
\text { above } 3 \mathrm{SD} \text { or less than } \\
-6 \mathrm{SD}\end{array}$ & $\begin{array}{l}\text { (1) Educational } \\
\text { achievement; (2) long- } \\
\text { term social outcomes }\end{array}$ \\
\hline $\begin{array}{l}\text { Lindström } \\
\text { et al. [20] }\end{array}$ & Sweden & $\begin{array}{l}\text { Retrospective cohort } \\
\text { population based }\end{array}$ & $\begin{array}{l}1973- \\
1979\end{array}$ & $23-29$ & $\begin{array}{l}37-38 \text { weeks } \\
\text { GA } \\
n=71,837\end{array}$ & $\begin{array}{l}\text { 39-41 weeks } \\
\text { GA } \\
n=450,165\end{array}$ & $\begin{array}{l}\text { At least } 1 \text { reported } \\
\text { malformation, BW } \\
\text { above } 3 \mathrm{SD} \text { or less than } \\
-6 \mathrm{SD} \text {, improbable } \\
\text { measures of length in } \\
\text { relation to BW }\end{array}$ & $\begin{array}{l}\text { Psychiatric and addictive } \\
\text { disorders defined } \\
\text { by using ICD-9 and } \\
\text { ICD-10 }\end{array}$ \\
\hline $\begin{array}{l}\text { Yang et al. } \\
{[21]}\end{array}$ & Canada & Prospective cohort & $\begin{array}{l}1996- \\
1997\end{array}$ & 6.5 & $\begin{array}{l}37-38 \text { weeks } \\
\text { GA } \\
n=2,569\end{array}$ & $\begin{array}{l}\text { 39-41 weeks } \\
\text { GA } \\
n=16,074\end{array}$ & $\mathrm{BW}<2,500 \mathrm{~g}$ & $\begin{array}{l}\text { Cognitive ability } \\
\text { measured by the } \\
\text { Wechsler Abbreviated } \\
\text { Scales of Intelligence }\end{array}$ \\
\hline $\begin{array}{l}\text { Moster et al. } \\
{[22]}\end{array}$ & Norway & $\begin{array}{l}\text { Retrospective cohort } \\
\text { population based }\end{array}$ & $\begin{array}{l}1967- \\
2001\end{array}$ & $\begin{array}{l}\text { At least } \\
4\end{array}$ & $\begin{array}{l}37-38 \text { weeks } \\
\text { GA } \\
n=218,911\end{array}$ & $\begin{array}{l}39-41 \text { weeks } \\
\text { GA } \\
n=1,215,350\end{array}$ & $\begin{array}{l}\text { BW above } 3 \text { SD, } \\
\text { registered congenital } \\
\text { anomalies, multiple } \\
\text { births }\end{array}$ & $\begin{array}{l}\text { Cerebral palsy diagnosed } \\
\text { by ICD-9 or } \\
\text { ICD- } 10\end{array}$ \\
\hline
\end{tabular}

$\mathrm{GA}=$ Gestational age; $\mathrm{BW}=$ birthweight $\mathrm{SD}=$ standard deviation; $\mathrm{ICD}=$ International Classification of $\mathrm{Disease}$. 
Table 2. Quality assessment of included studies

\begin{tabular}{|c|c|c|c|c|c|c|c|c|c|c|c|}
\hline $\begin{array}{l}\text { Summary of quality } \\
\text { assessment }\end{array}$ & $\begin{array}{l}\text { Eide } \\
\text { et al. [13] }\end{array}$ & $\begin{array}{l}\text { Quigley } \\
\text { et al. [14] }\end{array}$ & $\begin{array}{l}\text { Kirkegaard } \\
\text { et al. [15] }\end{array}$ & $\begin{array}{l}\text { MacKay } \\
\text { et al. [16] }\end{array}$ & $\begin{array}{l}\text { Lindström } \\
\text { et al. [17] }\end{array}$ & $\begin{array}{l}\text { Ekeus } \\
\text { et al. [18] }\end{array}$ & $\begin{array}{l}\text { Lindström } \\
\text { et al. [19] }\end{array}$ & $\begin{array}{l}\text { Lindström } \\
\text { et al. [20] }\end{array}$ & $\begin{array}{l}\text { Yang } \\
\text { et al. [21] }\end{array}$ & $\begin{array}{l}\text { Mathiasen } \\
\text { et al. [11] }\end{array}$ & $\begin{array}{l}\text { Moster } \\
\text { et al. [22] }\end{array}$ \\
\hline $\begin{array}{l}\text { Data source }[7,9] \\
\text { There are sufficient details } \\
\text { on the data source to limit } \\
\text { selection and measurement } \\
\text { bias }\end{array}$ & yes & yes & yes & yes & yes & yes & yes & yes & yes & yes & yes \\
\hline $\begin{array}{l}\text { Study participation [8] } \\
\text { The study sample represents } \\
\text { the population of interest on } \\
\text { key characteristics, sufficient } \\
\text { to limit potential bias in the } \\
\text { results }\end{array}$ & partly & yes & yes & yes & yes & partly & yes & yes & partly & yes & partly \\
\hline $\begin{array}{l}\text { Study attrition [8] } \\
\text { Loss to follow-up (from } \\
\text { sample to study population) } \\
\text { is not associated with key } \\
\text { characteristics, sufficient to } \\
\text { limit potential bias }\end{array}$ & partly & partly & partly & yes & unsure $^{a}$ & no & unsure $^{\mathrm{a}}$ & unsure $^{\mathrm{a}}$ & yes & unsure $^{a}$ & unsure $^{\mathrm{a}}$ \\
\hline $\begin{array}{l}\text { Prognostic factor } \\
\text { measurement [8] } \\
\text { The prognostic factor of } \\
\text { interest is adequately } \\
\text { measured in study } \\
\text { participants to sufficiently } \\
\text { limit potential bias }\end{array}$ & yes & yes & yes & yes & yes & yes & yes & yes & yes & yes & yes \\
\hline $\begin{array}{l}\text { Outcome measurement [8] } \\
\text { The outcome of interest is } \\
\text { adequately measured in } \\
\text { study participants to } \\
\text { sufficiently limit potential } \\
\text { bias }\end{array}$ & yes & yes & partly & yes & yes & yes & yes & yes & yes & yes & yes \\
\hline $\begin{array}{l}\text { Confounding measurement } \\
\text { and account [8] } \\
\text { Important potential } \\
\text { confounders are } \\
\text { appropriately accounted for, } \\
\text { limiting potential bias with } \\
\text { respect to the prognostic } \\
\text { factor of interest }\end{array}$ & partly & partly & yes & yes & yes & yes & yes & yes & yes & yes & yes \\
\hline $\begin{array}{l}\text { Analysis [8] } \\
\text { The statistical analysis is } \\
\text { appropriate for the design of } \\
\text { the study, limiting potential } \\
\text { for presentation of invalid } \\
\text { results }\end{array}$ & yes & yes & partly & yes & yes & yes & yes & yes & yes & yes & yes \\
\hline
\end{tabular}

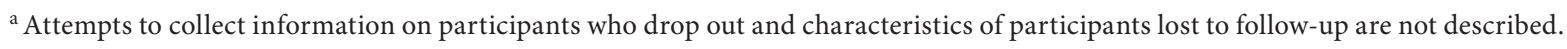

confounders into account, the risks of having several schooling problems for ETIs decreased [14-16]. However, the significance remained even after an extensive adjustment was performed.

\section{Behavior and Emotional Status}

Lindström et al. [17] carried out a population-based cohort study to analyze the effect of preterm birth on the risk of attention-deficit/hyperactivity disorder (ADHD) among school age children. The adjusted risk of receiving ADHD medications was significantly higher among ETIs than that among infants born at 39-41 weeks of gestational age (GA). However, the actual risk for ETIs might be underestimated due to the fact that receiving ADHD medications indicates severe cases of ADHD, and it is highly probable that much milder cases may be left unac- 
Table 3. A summary of results of studies

\begin{tabular}{llllll}
\hline Study & $\begin{array}{l}\text { Age at } \\
\text { assessment } \\
\text { years }\end{array}$ & Participants Adverse outcomes & Unadjusted result & Confounding factors Adjusted result \\
\hline
\end{tabular}

School outcomes

$\begin{array}{lll}\text { Quigley } & 5 & 37-38 \text { vs. } \\ \text { et al. [14] } & & 39-41 \text { weeks }\end{array}$

Not in good level of overall RR (95\% CI): achievement $\quad 1.09(1.03,1.15)$

Not working securely $\quad$ RR $(95 \% \mathrm{CI})$ : in math
$1.19(1.09,1.3)$
Sex, ethnicity, birth order, $\quad$ aRR $(95 \% \mathrm{CI})$ : multiple birth, breast $\quad 1.05(1.00,1.11)$ feeding duration, month $\quad$ aRR $(95 \% \mathrm{CI})$ : of birth, mother's age, marital status, education, social class and language

\begin{tabular}{llll}
\hline Kirkegaard & 10 & $37-38$ vs. & Having difficulty in reading OR: 1.55 \\
et al. [15] & & $39-40$ weeks & Having difficulty in spelling OR: 1.54 \\
& & Having difficulty in & OR: 0.82
\end{tabular}
mathematics
Gender, breastfeeding, and parental educational level
aOR (95\% CI):

$1.46(1.01,2.10)$ aOR (95\% CI)

$1.47(1.01,2.14)$ aOR (95\% CI): $0.71(0.36,1.41)$

MacKay et al. [16]

4-19

19

\author{
37 vs. 40 weeks \\ 38 vs. 40 weeks \\ 39 vs. 40 weeks
}

OR $(95 \% \mathrm{CI})$ :
37 weeks: $1.43(1.34$,
$1.52)$
38 weeks: $1.24(1.18$,
$1.29)$
39 weeks: $1.09(1.05$
$1.14)$

1.14)

\begin{tabular}{lllll}
\hline $\begin{array}{l}\text { Lindström } \\
\text { et al. [19] }\end{array}$ & $23-29$ & $\begin{array}{l}37-38 \text { vs. } \\
39-41 \text { weeks }\end{array}$ & $\begin{array}{l}\text { Missing or not completing } \\
\text { the compulsory 9 years of } \\
\text { school }\end{array}$ & $\begin{array}{l}\text { Prevalence (\%): } \\
0.6 \text { vs. } 0.4 \%\end{array}$ \\
\hline $\begin{array}{l}\text { Mathiasen } \\
\text { et al. [11] }\end{array}$ & $18-19$ & $\begin{array}{l}37-41 \text { weeks } \\
\text { no comparison } \\
\text { was made }\end{array}$ & $\begin{array}{l}\text { Failing to pass the exam } \\
\text { after 9 years of } \\
\text { compulsory school }\end{array}$ & $\begin{array}{l}\text { Prevalence (\%): } \\
37 \text { weeks: } 11.9 \%, 38 \\
\text { weeks: } 10.5 \%, 39 \\
\text { weeks: } 9.4 \%, 40 \text { weeks: } \\
8.7 \%, 41 \text { weeks: } 8.5 \%{ }^{\text {a }}\end{array}$
\end{tabular}

38 weeks: 1.19

\begin{tabular}{lll}
\hline $\begin{array}{l}\text { Neurodevelopment } \\
\text { Mathiasen }\end{array} 18-19$ & $\begin{array}{l}\text { 37-41 weeks } \\
\text { no comparison } \\
\text { et al. [11] }\end{array}$ & Cerebral palsy \\
& &
\end{tabular}

\begin{tabular}{|c|c|c|c|}
\hline $\begin{array}{l}\text { Moster } \\
\text { et al. [22] }\end{array}$ & 4 & $\begin{array}{l}37 \text { vs. } 40 \text { weeks } \\
38 \text { vs. } 40 \text { weeks } \\
39 \text { vs. } 40 \text { weeks }\end{array}$ & Cerebral palsy \\
\hline
\end{tabular}

Sex, marital status, parity, birth weight centile, induction of labor, mode of delivery, year of delivery, previous spontaneous and therapeutic deliveries, 5-min Apgar score

aOR (95\% CI):

37 weeks: 1.36

(1.27, 1.45)

(1.14, 1.25)

39 weeks: 1.09

39 weeks: 1.09
$(1.04,1.14)$

Not reported

Prevalence (\%): 37
weeks: $0.6 \%, 38$ weeks:

Not reported

$0.4 \%$, 39 weeks: $0.3 \%$,

40 weeks: $0.2 \%$,

41 weeks: $0.2 \%$

RR (95\% CI):

37 weeks: $1.9(1.6,2.4)$

38 weeks: $1.3(1.1,1.5)$

39 weeks: $1.1(1.0,1.3)$

40 weeks: 1.0

$\begin{array}{ll}\begin{array}{l}\text { Year of birth, infant sex, } \\ \text { maternal age, presence or } \\ \text { absence of a partner, } \\ \text { educational level of the } \\ \text { mother and father, } \\ \text { immigrant status of the } \\ \text { parents }\end{array} & \begin{array}{l}\text { aRR was } \\ \text { crude RR }\end{array} \\ \begin{array}{l}\text { Not reported } \\ \text { Maternal age, maternal }\end{array} \\ \begin{array}{l}\text { education, parity, height } \\ \text { and body mass index }\end{array} & \begin{array}{l}\text { Mean difference } \\ (95 \% \text { CI }):-0.06 \\ (-0.07,-0.05)\end{array} \\ \begin{array}{l}\text { Year of birth, age at } \\ \text { conscription, conscription } \\ \text { office, SES, maternal }\end{array} & \begin{array}{l}\text { Mean difference } \\ (95 \% \text { CI }):-0.03 \\ (-0.06,0.00)\end{array}\end{array}$

education, marital status of mother, maternal age, and parity Above factors plus SGA and low Apgar score
Mean difference (95\% CI): -0.02 $(-0.06,-0.01)$ 
Table 3 (continued)

\begin{tabular}{|c|c|c|c|c|c|c|}
\hline Study & $\begin{array}{l}\text { Age at } \\
\text { assessment } \\
\text { years }\end{array}$ & Participants & Adverse outcomes & Unadjusted result & Confounding factors & Adjusted result \\
\hline $\begin{array}{l}\text { Yang } \\
\text { et al. [21] }\end{array}$ & 6.5 & $\begin{array}{l}37 \text { vs. } 39-41 \\
\text { weeks } \\
38 \text { vs. } 39-41 \\
\text { weeks }\end{array}$ & Intelligence score & $\begin{array}{l}\text { Mean difference } \\
\text { (95\% CI): } 37 \text { weeks: } \\
-2.6(-3.7,-1.4) \\
38 \text { weeks: }-0.6(-1.1 \text {, } \\
-0.01)\end{array}$ & $\begin{array}{l}\text { BW for GA, sex, maternal } \\
\text { height and age, smoking } \\
\text { and drinking during } \\
\text { pregnancy } \\
\text { Above factors plus } \\
\text { parental marital status, } \\
\text { number of children in } \\
\text { household, parental } \\
\text { education and occupation }\end{array}$ & $\begin{array}{l}\text { Mean difference } \\
(95 \% \text { CI): } \\
37 \text { weeks: }-2.4 \\
(-3.5,-1.3) \\
38 \text { weeks: }-0.5 \\
(-1.0,-0.1) \\
\text { Mean difference } \\
\text { (95\% CI): } \\
37 \text { weeks: }-1.7 \\
(-2.7,-0.7) \\
38 \text { weeks: }-0.4 \\
(-1.1,-0.2)\end{array}$ \\
\hline \multicolumn{7}{|c|}{ Behavior and emotional status } \\
\hline $\begin{array}{l}\text { Lindström } \\
\text { et al. [17] }\end{array}$ & $6-19$ & $\begin{array}{l}37-38 \text { vs. } \\
39-41 \text { weeks }\end{array}$ & $\begin{array}{l}\text { Receiving at least } 1 \\
\text { prescription of ADHD } \\
\text { medication }\end{array}$ & $\begin{array}{l}\text { OR }(95 \% \text { CI }): \\
1.2(1.1,1.3)\end{array}$ & $\begin{array}{l}\text { Year of birth, gender, } \\
\text { residence, birth order, } \\
\text { maternal age, maternal } \\
\text { education, single } \\
\text { parenthood, public } \\
\text { welfare, maternal } \\
\text { smoking, maternal/ } \\
\text { paternal, psychiatric/ } \\
\text { addictive disorders, low } \\
\text { Apgar score and SGA }\end{array}$ & $\begin{array}{l}\mathrm{aOR}(95 \% \mathrm{CI}) \text { : } \\
1.1(1.1,1.2)\end{array}$ \\
\hline $\begin{array}{l}\text { Lindström } \\
\text { et al. [20] }\end{array}$ & $23-29$ & $\begin{array}{l}37-38 \text { vs. } \\
39-41 \text { weeks }\end{array}$ & $\begin{array}{l}\text { Having been discharged } \\
\text { from hospital at least once } \\
\text { with a main psychiatric } \\
\text { diagnosis }\end{array}$ & $\begin{array}{l}\text { HR }(95 \% \text { CI): } \\
1.11(1.05,1.16)\end{array}$ & $\begin{array}{l}\text { Age, gender, housing, } \\
\text { social welfare, SES, } \\
\text { parental psychiatric } \\
\text { disorder, low Apgar score } \\
\text { and SGA }\end{array}$ & $\begin{array}{l}\text { aHR (95\% CI): } \\
1.06(1.01,1.12)\end{array}$ \\
\hline
\end{tabular}

${ }^{\text {a }}$ Data not published.

counted for [17]. In another cohort from the same country, Lindström et al. [20] explored the hazard ratio of having a psychiatric disorder for ETIs. The risk was reduced from $1.11(95 \%$ CI $1.05,1.16)$ to 1.06 (95\% CI 1.01, 1.12) after taking perinatal factors, SES and the home environment into account [20].

\section{Long-Term Social Outcome}

A large-scale cohort study analyzed the effect of GA on disability and vocational success among young adults [19]. Potential confounding factors included maternal factors, SES and single-parent household. In comparison to adults born at 39-41 gestational weeks, those born early term carried significantly increased risk for disability [adjusted RR 1.26 (95\% CI 1.17, 1.35)]. However, the chance of getting employed [adjusted RR 0.99 (95\% CI $0.98,1.02)$ ] and the mean net salary (mean difference: -6 EUR; $p=0.907$ ) were identical between the two groups, suggesting the adverse effect of being born early term on certain social outcomes might be attenuated by long-term postnatal environments [19].

\section{Discussion}

ETIs are a newly defined subgroup of neonates who are beginning to arouse attention due to their suboptimal long-term outcomes shown by emerging evidence. In this systematic review of long-term developmental outcomes of ETIs, none of the 11 retrieved articles focused solely on ETIs, highlighting a paucity of relative literature. Although wide heterogeneity existed among included studies, all authors reported a consistent trend of poorer outcomes among ETIs than that among infants born at 3941 weeks of GA. It should be noted some cohorts were born before ultrasound became a routine procedure to 


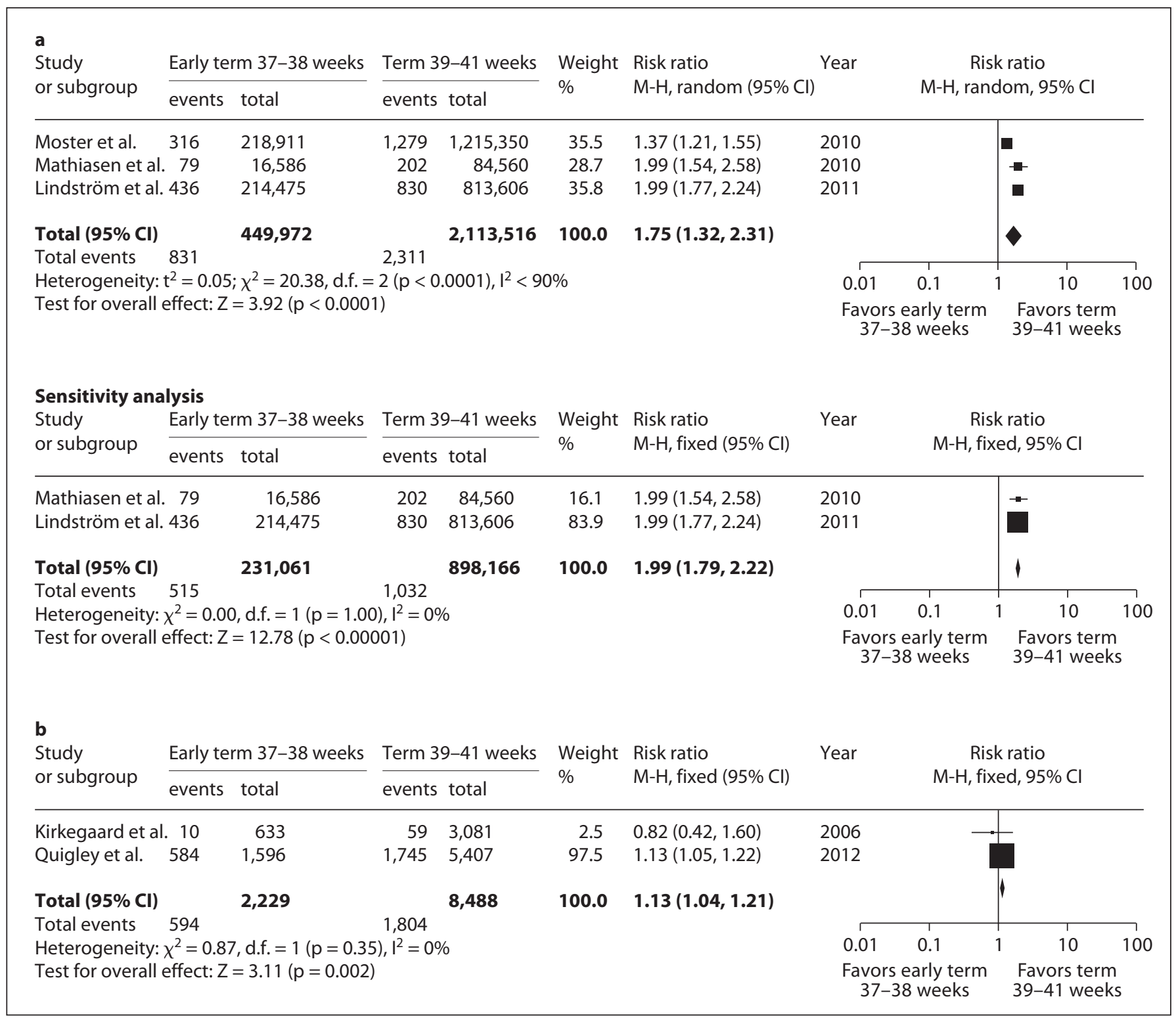

Fig. 2. Meta-analyses of the risk of adverse long-term outcomes for ETIs compared with term infants born at 39-41 gestational weeks. a Forest plot of the risk and 95\% CI of cerebral palsy for ETIs. b Forest plot of the risk and $95 \%$ CI of mathematical difficulties for ETIs.

measure GA, and a considerable number of individuals born term were miscoded as preterm, which may lead to underestimation of the effect of being born early term on long-term risks [11, 13, 17-20,22]. Compared with infants of 39-41 gestational weeks, a significant increase in the risk of $\mathrm{CP}$ and mathematical difficulties among ETIs was shown by our meta-analyses.

The traditional assumption of gestation to be a dichotomous developmental course has been challenged as mounting evidence demonstrates that the dose-dependent effect of gestation on neonatal outcomes exists not only across the spectrum of preterm but also within the boundary of term $[1,2]$. Over the last several years, risks posed to late preterm infants, a marginally term group, have been sufficiently realized, and much effort has been dedicated to prevent late-term deliveries $[25,26]$. Recently, ETIs were defined to raise concerns for infants born at the more optimistic stage of term, stressing the fact that 
gestation is a biological continuum, and any classification tends to be arbitrary [3]. A reverse J-shaped relationship between the risk of suboptimal outcomes and GA across the whole gestational spectrum indicates that the optimal time of gestation occurs somewhere between 39 and 41 completed weeks, a much narrower interval than the historically held 5-week term period $[16,22]$. A delivery occurring earlier or later may increase the risk of adverse outcomes.

Our results demonstrated that each additional week of gestation prior to 39 gestational weeks was accompanied by increased intelligence scores and a reduced risk of CP. The gradient suggests that the relation between GA and brain development is present across the whole gestation and not confined solely to preterm infants. Active myelination and significant growth in various kinds of brain cells are observed between 34 and 40 weeks of GA, which contributes to the $50 \%$ increase in cortical volume and the $25 \%$ increase in cerebellar development [27]. Inge et al. [28] assessed the brain volumes of normally developing children when they were 9 years old, finding that ETIs had a smaller cerebellar volume than their counterparts who were born at 39-41 weeks' gestation even after adjusting for birthweight, age, sex and head size. Davis et al. [29] found that among healthy term infants without any perinatal risk factors such as intraventricular hemorrhage and periventricular leukomalacia, a longer gestation was associated with an increase in gray matter density of certain brain regions. These data suggest that shortened gestations even within the spectrum of term can interrupt the process of brain maturity. Additionally, perinatal risk factors such as neonatal morbidities and medical treatments can compound existing biological vulnerabilities and impede postnatal brain development [29]. Apart from obvious disabilities like CP, early term births are at risk of subtle developmental impairments such as schooling problems, behavioral and emotional problems, and social disadvantages, the underlying mechanisms being largely unknown [11, 13-16, 19]. It is noted that associations between GA and adverse longterm outcomes are attenuated with adjustment for potential confounders, most of which are associated with parental characteristics and housing conditions, suggesting that postnatal environments may compensate for neonatal disadvantages. Therefore, a long-term follow-up combined with appropriate interventions may help identify and alleviate potential problems encountered by ETIs during their process of development.

The risk of bias is considered to be higher in a systematic review of non-randomized studies due to the lack of randomization and loss to follow-up. The strength of this review is that most included studies are high-quality population-based cohort studies, which is considered helpful in reducing selection bias. Even though current evidence generally supports that ETIs have an increased risk of suboptimal long-term development, a conclusive statement cannot be reached given the limitations of included studies and the wide heterogeneity among them. Although extensive adjustments are conducted in included studies, most of the participants retrospectively enrolled were born prior the last decade or even as much as 4 decade ago, during which time the medical technology evolved rapidly, and the care provided to ETIs may not be uniform; therefore, the chance of confounding bias remains high. Additionally, the prevalence rate of an adverse outcome among ETIs is considerably low when compared with that among infants born more preterm; a large sample of population might be needed to detect an effect. Therefore, reporting biases will arise as small-size studies with negative results are not likely to be reported or published. As demonstrated in our review, wide variations exist in population size, demographic characteristics, follow-up period and outcomes of interest. The results of our meta-analysis should be interpreted with caution due to the limited number of included studies. Standardization of future study protocols is needed, so that more studies can be quantitatively assessed and the risk of suboptimal outcomes under different categories can be adequately evaluated for ETIs.

\section{Conclusion}

ETIs are a subgroup of neonates assuming great importance in public health. However, the long-term risks associated with early term births have not been well understood due to the heterogeneity and paucity of current data. A complex series of factors can impact the trajectory of development in ETIs, which needs to be clarified by further studies with an adequate population size and a standardized study design. As evidence accumulates, the management of ETIs can be guided and optimized.

\section{Acknowledgement}

We would like to thank Dr. Mathiasen for providing additional data of their published study. 


\section{References}

$>1$ Fleischman AR, Oinuma M, Clark SL: Rethinking the definition of 'term pregnancy'. Obstet Gynecol 2010;116:136-139.

-2 Saigal S, Doyle LW: An overview of mortality and sequelae of preterm birth from infancy to adulthood. Lancet 2008;371:261-269.

$\checkmark 3$ Engle WA: Morbidity and mortality in late preterm and early term newborns: a continuum. Clin Perinatol 2011;38:493-516.

$\checkmark 4$ Davidoff MJ, Dias T, Damus K, Russell R, Betteqowda VR, Dolan S, Schwarz R, Green NS, Petrini J: Changes in the gestational age distribution among US singleton births; impact on rates of late preterm birth, 1992 to 2002. Semin Perinatol 2006;30:8-15.

5 Hankins GDV, Clark S, Munn MB: Cesarean section on request at 39 weeks: impact on shoulder dystocia, fetal trauma, neonatal encephalopathy, and intrauterine fetal demise. Semin Perinatol 2006;30:276-287.

$\checkmark 6$ Engle WA, Kominiarek MA: Late preterm infants, early term infants, and timing of elective deliveries. Clin Perinatol 2008;35: 325-341.

$\checkmark 7$ Mcgowan JE, Alderdice FA, Holmes VA, Johnston L: Early childhood development of late-preterm infants: a systematic review. Pediatrics 2011;127:1111-1124.

$>8$ Hayden JA, Cote P, Bombardier C: Evaluation of the quality of prognosis studies in systematic reviews. Ann Intern Med 2006;144: 427-437.

$\checkmark 9$ Motheral B, Brooks J, Clark MA, Crown WH, Davey P, Hutchins D, Martin BC, Stang P: A checklist for retrospective database studies - report of the ISPOR Task Force on Retrospective Databases. Value Health 2003 6:90-97.

10 Higgins J, Green S: Cochrane Handbook for Systematic Reviews of Interventions. Chichester, John Wiley \& Sons, 2008, pp 190-191.
11 Mathiasen R, Hansen BM, Andersen AM, Forman JL, Greisen G: Gestational age and basic school achievements: a national followup study in Denmark. Pediatrics 2010; 126:e1553-e1561.

12 Wolke D, Schulz J, Meyer R: Entwicklung slangzeitfolgen bei ehemaligen, sehr unreifen Frühgeborenen Bayerische Entwicklungsstudie. Monatsschr Kinderheilkd 2001;(suppl 1):S53-S61.

13 Eide MG, Oyen N, Skjaerven R, Bjerkedal T: Associations of birth size, gestational age, and adult size with intellectual performance: evidence from a cohort of Norwegian men. Pediatr Res 2007;62:636-642.

14 Quigley MA, Poulsen G, Boyle E, Wolke D, Field D, Alfirevic Z, Kurinczuk JJ: Early term and late preterm birth are associated with poorer school performance at age 5 years: a cohort study. Arch Dis Child Fetal Neonatal Ed 2012;97:F167-F173.

15 Kirkegaard I, Obel C, Hedegaard M, Henriksen TB: Gestational age and birth weight in relation to school performance of 10 -yearold children: a follow-up study of children born after 32 completed weeks. Pediatrics 2006;118:1600-1606.

16 MacKay DF, Smith GC, Dobbie R, Pell JP: Gestational age at delivery and special educational need: retrospective cohort study of 407,503 schoolchildren. PLoS Med 2010; 7:e1000289.

-17 Lindström K, Lindblad F, Hjern A: Preterm birth and attention-deficit/hyperactivity disorder in schoolchildren. Pediatrics 2011; 127:858-865.

18 Ekeus C, Lindström K, Lindblad F, Rasmussen F, Hjern A: Preterm birth, social disadvantage, and cognitive competence in Swedish 18- to 19-year-old men. Pediatrics 2010 125:e67-e73.

19 Lindström K, Winbladh B, Haglund B, Hjern A: Preterm infants as young adults: a Swedish national cohort study. Pediatrics 2007; 120:70-77.
20 Lindström K, Lindblad F, Hjern A: Psychiatric morbidity in adolescents and young adults born preterm: a Swedish national cohort study. Pediatrics 2009;123:e47-e53.

-21 Yang S, Platt RW, Kramer MS: Variation in child cognitive ability by week of gestation among healthy term births. Am J Epidemiol 2010;171:399-406

-22 Moster D, Wilcox AJ, Vollset SE, Markestad T, Lie RT: Cerebral palsy among term and postterm births. JAMA 2010;304:976-982.

23 Shevell MI: Present conceptualization of early childhood neurodevelopmental disabilities. J Child Neurol 2010;25:120-126.

$>24$ Wolke D: Psychological development of prematurely born children. Arch Dis Child 1998;78:567-570

25 Barros MC, Mitsuhiro S, Chalem E, Laranjeira RR, Guinsburg R: Neurobehavior of late preterm infants of adolescent mothers. Neonatology 2011;99:133-139.

26 Raju TN, Higgins RD, Stark AR, Leveno KJ: Optimizing care and outcome for late-preterm (near-term) infants: a summary of the workshop sponsored by the National Institute of Child Health and Human Development. Pediatrics 2006;118:1207-1214.

27 Arpino C, Compagnone E, Montanaro ML, Cacciatore D, De Luca A, Cerulli A, Di Girolamo S, Curatolo P: Preterm birth and neurodevelopmental outcome: a review. Childs Nerv Syst 2010;26:1139-1149.

28 Inge LC,van Soelen IL, Brouwer RM, Peper JS, van Beijsterveldt TC, van Leeuwen M, de Vries LS, Kahn RS, Hulshoff Pol HE, Boomsma DI: Effects of gestational age and birth weight on brain volumes in healthy 9 yearold children. J Pediatr 2010;156:896-901.

29 Davis EP, Buss C, Muftuler LT, Head K, Hasso A, Wing DA, Hobel C, Sandman CA: Children's brain development benefits from longer gestation. Front Psychol 2011;2:1. 